Analele Universităţii de Vest,

Timişoara

Seria Matematică - Informatică

LII, 1, (2014), 65- 79

\title{
Some Simplicial Complexes of Universal Osborn Loops
}

\author{
Jaíyéọlá Tèmítộpẹ́ Gbọ́láhàn
}

To Prof. Dora Nkem Akunyili (July 14, 1954 - June 7, 2014)

\begin{abstract}
A loop is shown to be a universal Osborn loop if and only if it has a particular simplicial complex. A loop is shown to be a universal Osborn loop and obeys two new identities if and only if it has another particular simplicial complex. A universal Osborn loop and four of its isotopes are shown to form a rectangular pyramid in a 3 -dimensional space.
\end{abstract}

AMS Subject Classification (2000). 20N02; 20N05

Keywords. Osborn loops, universality, left universality, right universality

\section{Introduction and Preliminaries}

A loop is called an Osborn loop if it obeys any of the two identities below.

$$
\begin{aligned}
& \mathrm{OS}_{3}:(x \cdot y z) x=x y \cdot\left[\left(x^{\lambda} \cdot x z\right) \cdot x\right] \\
& \mathrm{OS}_{5}:(x \cdot y z) x=x y \cdot\left[\left(x \cdot x^{\rho} z\right) \cdot x\right]
\end{aligned}
$$

For a comprehensive introduction to Osborn loops and its universality, and a detailed literature review on it, readers should check Jaiyéolá, Adéníran and Sòlárìn [3] and Jaiyéolá [4]. In this present paper, we shall follow the style and notations used in Jaiyéọlá, Adéníran and Sòlárìn [3] and Jaiyéọlá [4]. The only concepts and notions which will be introduced here are those that were not defined in Jaiyéọlá, Adéníran and Sòlárìn [3] and Jaiyéọlá [4]. 
Definition 1.1. Let $(L, \cdot)$ be a loop and $U, V, W \in S Y M(L, \cdot)$.

1. If $(U, V, W) \in A U T(L, \cdot)$ for some $V, W$, then $U$ is called autotopic.

2. If $(U, V, W) \in A U T(L, \cdot)$ such that $W=U, V=I$, then $U$ is called $\lambda$-regular.

3. If $(U, V, W) \in A U T(L, \cdot)$ such that $U=I, W=V$, then $V$ is called $\rho$-regular.

Stein [5] and Drisko [2] while considering the action of isotopisms and autotopisms of loops, found it convenient to think of a loop $Q=(Q, \cdot, \backslash, /)$ in terms of the set $T_{Q}$ of all ordered triples $(x, y, z)$ of elements of $Q$ such that $x \cdot y=z$. An isotopism $(\alpha, \beta, \gamma)$ from $G$ to $H$ takes $(x, y, z) \in T_{G}$ to $(x \alpha, y \beta, z \gamma) \in T_{H}$. We shall adopt his conventions at some points in time. We shall denote by $[\alpha, \beta]$, the commutator of any $\alpha, \beta \in S Y M(G, \cdot)$.

Let $(Q, \cdot, \backslash, /)$ be a loop, then we shall be making use of the following notations for principal isotopes of $(Q, \cdot)$.

- $\left(Q, *_{0}\right)$ represents $Q_{x, v}$;

- $\left(Q, \circ_{0}\right)$ represents $Q_{u, \phi_{0}(x, u, v)}, \phi_{0}(x, u, v)=(u \backslash([(u v) /(u \backslash(x v))] v))$;

- $\left(Q, \circ_{1}\right)$ represents $Q_{u,[u \backslash(x v)]}$;

- $\left(Q, *_{1}\right)$ represents $Q_{\phi_{1}(x, u, v), v}, \phi_{1}(x, u, v)=(u \backslash([(u v) /(u \backslash(x v))] v))$ for all $x, u, v \in Q$;

- $\left(Q, \circ_{2}\right)$ represents $Q_{x, \phi_{2}(x, u, v)}, \phi_{2}(x, u, v)=(u \backslash[(u / v)(u \backslash(x v))])$;

- $\left(Q, \circ_{3}\right)$ represents $Q_{[x \cdot u \backslash v] / v,[u \backslash(x v)]}$;

- $\left(Q, *_{2}\right)$ represents $Q_{u, e}$;

- $\left(Q, *_{3}\right)$ represents $Q_{e, v}$.

Let $(G, \cdot)$ be a loop and let

$$
B S_{2}(G, \cdot)=\{\theta \in S Y M(G): G(a, b) \stackrel{\theta}{\cong} G(c, d) \text { for some } a, b, c, d \in G\}
$$

As shown in Bryant and Schneider [1], $B S_{2}(G, \cdot)$ forms a group for a loop $(G, \cdot)$ and it shall be called the second Bryant-Schneider group ( $2^{\text {nd }}$ BSG) of the loop.

Consider the following two notions in algebraic topology. 
Definition 1.2. Let $V_{Q}$ be a set of isotopes of a loop $(Q, \cdot)$ and let $S_{Q} \subseteq 2^{V_{Q}}$ such that $\phi \in S_{Q}$. If $S_{Q}$ is a topology on $V_{Q}$, then it is called the topology of isotopes of the loop $Q$ and the pair $\left(V_{Q}, S_{Q}\right)$ is called a topological space of isotopes of $Q$ if $\left(V_{Q}, S_{Q}\right)$ is a topological space.

Based on the above notion of topological space of isotopes of a loop, the following facts are direct consequences.

Lemma 1.1. Let $(Q, \cdot)$ be a loop and let $V_{Q}$ be the set of isotopes of $Q$. Then, $\left(V_{Q}, \mathcal{Q}^{V_{Q}}\right)$ is a topological space of isotopes of $Q$.

Lemma 1.2. Let $(Q, \cdot)$ be a $G$-loop and let $V_{Q}$ be the set of isotopes of $Q$. Let $S_{Q}=\left\{X_{i}\right\}_{i \in \Omega} \subseteq 2^{V_{Q}}$ such that $\phi \in S_{Q}$ and $x_{i_{j}} \cong x_{i_{k}}$ for all $x_{i_{j}}, x_{i_{k}} \in X_{i}$. Then, $\left(V_{Q}, S_{Q}\right)$ is a topological space of isotopes of $Q$.

Corollary 1.3. Let $(Q, \cdot)$ be a CC-loop or VD-loop or K-loop or Buchsteiner loop or extra loop or group. Let $S_{Q}=\left\{X_{i}\right\}_{i \in \Omega} \subseteq \mathcal{Q}^{V_{Q}}$ such that $\phi \in S_{Q}$ and $x_{i_{j}} \cong x_{i_{k}}$ for all $x_{i_{j}}, x_{i_{k}} \in X_{i}$. Then, $\left(V_{Q}, S_{Q}\right)$ is a topological space of isotopes of $Q$.

Definition 1.3. A simplicial complex is a pair $(V, S)$ where $V$ is a set of points called vertices and $S$ is a given family of finite subsets, called simplexes, so that the following conditions are satisfied:

1. all points of $V$ are simplexes;

2. any non-empty subset of a simplex is a simplex.

A simplex consisting of $(n+1)$ points is called $n$-dimensional simplex.

Definition 1.4. Let $V_{Q}$ be a set of isotopes of a loop $(Q, \cdot)$ and let $S_{Q} \subseteq \mathcal{2}^{V_{Q}}$. If $K_{Q}=\left(V_{Q}, S_{Q}\right)$ is a simplicial complex, then $K_{Q}$ is called a trivial simplicial complex of isotopes of the loop $Q$.

Definition 1.5. Let $V_{Q}$ be a set of isotopes of a loop $(Q, \cdot)$ and let $S_{Q}=$ $\left\{X_{i}\right\}_{i \in \Omega} \subseteq \mathcal{Q}^{V_{Q}}$ such that $x_{i_{j}} \cong x_{i_{k}}$ for all $x_{i_{j}}, x_{i_{k}} \in X_{i}$. If $K_{Q}=\left(V_{Q}, S_{Q}\right)$ is a simplicial complex, then $K_{Q}$ is called a non-trivial simplicial complex of isotopes or simplicial complex of isotopes of the loop $Q$.

The facts below follow suite.

Lemma 1.4. Let $(Q, \cdot)$ be a loop and let $V_{Q}$ be the set of isotopes of $Q$. Then, $\left(V_{Q}, 2^{V_{Q}}\right)$ is a trivial simplicial complex of isotopes of $Q$. 
Lemma 1.5. Let $(Q, \cdot)$ be a $G$-loop and let $V_{Q}$ be the set of isotopes of $Q$. Let $S_{Q}=\left\{X_{i}\right\}_{i \in \Omega} \subseteq \mathcal{2}^{V_{Q}}$ such that $x_{i_{j}} \cong x_{i_{k}}$ for all $x_{i_{j}}, x_{i_{k}} \in X_{i}$. Then, $\left(V_{Q}, S_{Q}\right)$ is a simplicial complex of isotopes of $Q$.

Corollary 1.6. Let $(Q, \cdot)$ be a CC-loop or VD-loop or K-loop or Buchsteiner loop or extra loop or group. Let $S_{Q}=\left\{X_{i}\right\}_{i \in \Omega} \subseteq 2^{V_{Q}}$ such that $x_{i_{j}} \cong x_{i_{k}}$ for all $x_{i_{j}}, x_{i_{k}} \in X_{i}$. Then, $\left(V_{Q}, S_{Q}\right)$ is a simplicial complex of isotopes of $Q$.

Definition 1.6. Let $K=(V, S)$ and $K^{\prime}=\left(V^{\prime}, S^{\prime}\right)$ be two simplicial complexes. A simplicial map $f: K \rightarrow K^{\prime}$ is a set map $f: V \rightarrow V^{\prime}$ satisfying the property: for every simplex $x \in S$, the image $f(x) \in S^{\prime}$.

In this work, the notion of simplicial complex is used to characterize universal Osborn loops. The following results are important for the set objective.

Theorem 1.7. (Jaiyéolá , Adéníran and Sòlárin [3])

Let $Q=(Q, \cdot, \backslash, /)$ be a loop and $\gamma_{0}(x, u, v)=\mathbb{R}_{v} R_{[u \backslash(x v)]} \mathbb{L}_{u} L_{x}$ for all $x, u, v \in$ $Q$, then $\mathcal{Q}$ is a universal Osborn loop if and only if the commutative diagram

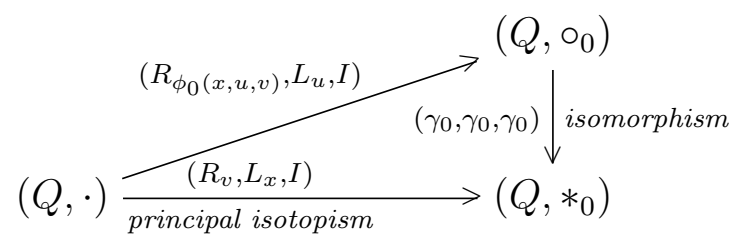

holds.

Theorem 1.8. (Jaíyéolá [4])

Let $\mathcal{Q}=(Q, \cdot, \backslash, /)$ be a loop and $\gamma_{1}(x, u, v)=\mathbb{R}_{v} R_{[u \backslash(x v)]} \mathbb{L}_{u} L_{x}$ for all $x, u, v \in$ $Q$, then $\mathcal{Q}$ is a universal Osborn loop if and only if the commutative diagram

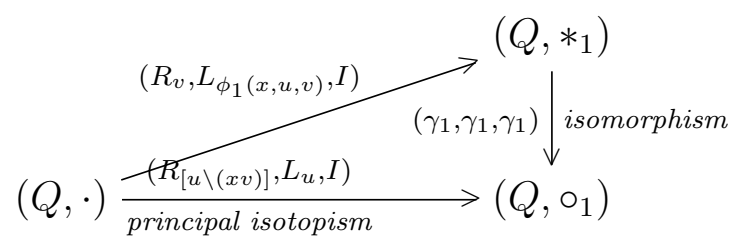

holds.

Theorem 1.9. (Jaiyéọlá, Adéníran and Sòlárin [3])

Let $\mathcal{Q}=(Q, \cdot, \backslash, /)$ be a loop and $\gamma_{0}(x, u, v)=\mathbb{R}_{v} R_{[u \backslash(x v)]} \mathbb{L}_{u} L_{x}$ for all $x, u, v \in$ $Q$, then $\mathcal{Q}$ is a universal Osborn loop implies the commutative diagram

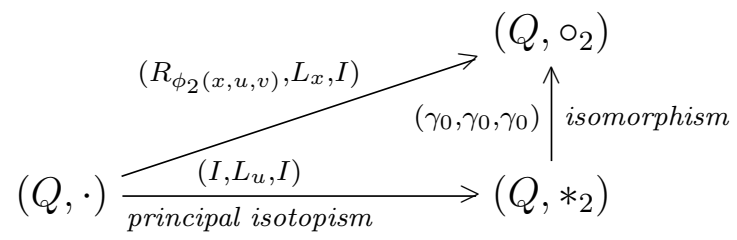

holds. 
Theorem 1.10. (Jaíyéọlá [4])

Let $\mathcal{Q}=(Q, \cdot, \backslash, /)$ be a loop and $\gamma_{1}(x, u, v)=\mathbb{R}_{v} R_{[u \backslash(x v)]} \mathbb{L}_{u} L_{x}$ for all $x, u, v \in$ $Q$, then $\mathrm{Q}$ is a universal Osborn loop implies the commutative diagram

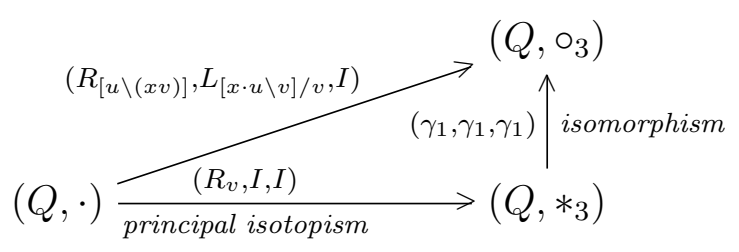

holds.

Lemma 1.11. (Drisko [2])

Let $\mathcal{Q}=(Q, \cdot, \backslash, /)$ be a loop. Then $Q_{f, g} \cong Q_{c, d}$ if and only if there exists $(\alpha, \beta, \gamma) \in \operatorname{AUT}(\mathcal{Q})$ such that $(f, g, f g)(\alpha, \beta, \gamma)=(c, d, c d)$.

Theorem 1.12. (Bryant and Schneider [1])

Let $(Q, \cdot, \backslash, /)$ be a quasigroup. If $Q_{a, b} \stackrel{I}{\cong} Q_{c, d}$ if and only if $c \cdot b, a \cdot d \in N_{\mu}\left(Q_{a, b}\right)$ and $a \cdot b=c \cdot d$.

\section{Main Results}

Theorem 2.1. Let $Q=(Q, \cdot, \backslash, /)$ be a universal Osborn loop. Then, the following are necessary and sufficient for each other.
1. $\left(Q, \circ_{0}\right) \stackrel{I}{\cong}\left(Q, \circ_{1}\right)$.
2. $\left(Q, *_{0}\right) \stackrel{I}{\cong}\left(Q, *_{1}\right)$.
3. $\mathcal{Q}$ is a boolean group.

Proof. By combining the commutative diagrams in Equation 1.3 and Equa- 
tion 1.4, we have the commutative diagram below.

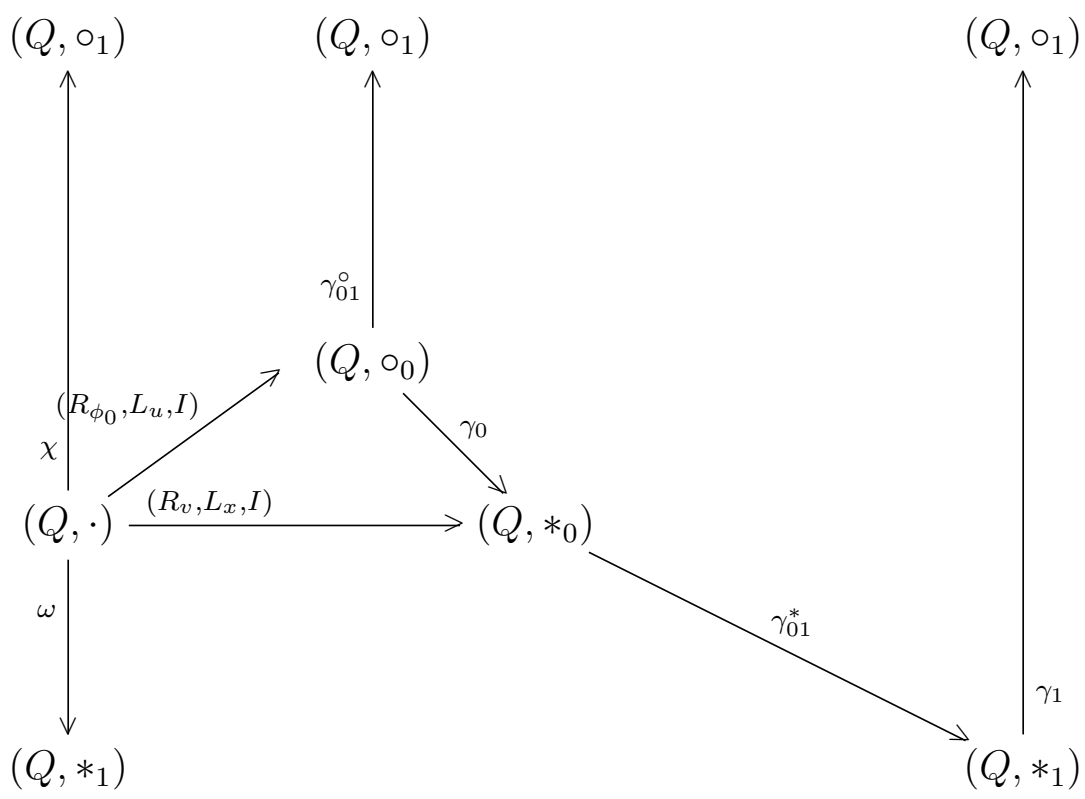

where $\chi=\left(R_{[u \backslash(x v)]}, L_{u}, I\right)$ and $\omega=\left(R_{v}, L_{\phi_{1}}, I\right)$. Let

$$
\left(Q, \circ_{0}\right) \underset{\text { isotopism }}{\stackrel{\left(\delta_{01}^{\circ}, \varepsilon_{01}^{\circ}, \pi_{01}^{\circ}\right)}{\longrightarrow}}\left(Q, \circ_{1}\right) .
$$

So, from Equation 2.1,

$$
\begin{gathered}
\left(R_{\phi_{0}(x, u, v)}, L_{u}, I\right)\left(\delta_{01}^{\circ}, \varepsilon_{01}^{\circ}, \pi_{01}^{\circ}\right)=\left(R_{[u \backslash(x v)]}, L_{u}, I\right) \Rightarrow \\
\left(R_{\phi_{0}(x, u, v)} \delta_{01}^{\circ}, L_{u} \varepsilon_{01}^{\circ}, \pi_{01}^{\circ}\right)=\left(R_{[u \backslash(x v)]}, L_{u}, I\right) \Leftrightarrow \\
R_{\phi_{0}(x, u, v)} \delta_{01}^{\circ}=R_{[u \backslash(x v)]}, L_{u} \varepsilon_{01}^{\circ}=L_{u} \text { and } \pi_{01}^{\circ}=I \Leftrightarrow \\
\delta_{01}^{\circ}=R_{\phi_{0}(x, u, v)}^{-1} R_{[u \backslash(x v)]}, \quad \varepsilon_{01}^{\circ}=L_{u}^{-1} L_{u}=I \text { and } \pi_{01}^{\circ}=I .
\end{gathered}
$$

Thus, $\left(Q, \circ_{0}\right) \cong\left(Q, \circ_{1}\right)$ iff $\delta_{01}^{\circ}=\varepsilon_{01}^{\circ}=I$ iff

$$
\begin{gathered}
R_{\phi_{0}(x, u, v)}^{-1} R_{[u \backslash(x v)]}=I \Leftrightarrow \phi_{0}(x, u, v)=[u \backslash(x v)] \\
(u \backslash([(u v) /(u \backslash(x v))] v))=[u \backslash(x v)] \Leftrightarrow x \backslash(u v)=u \backslash(x v) .
\end{gathered}
$$

Similarly, by using the procedure above, it can be shown that $\left(Q, *_{0}\right) \cong$ $\left(Q, *_{1}\right)$ iff $x \backslash(u v)=u \backslash(x v)$.

Keeping in mind that every Osborn loop of exponent 2 is an abelian group, hence, a Boolean group. This completes the proof.

Remark 2.1. It can be observed that in a universal Osborn loop $Q=$ $(Q, \cdot, \backslash, /)$ and for $\gamma_{0}(x, u, v)$ and $\gamma_{1}(x, u, v)$ of Theorem 1.7 and Theorem 1.8, 
$\gamma_{0}(x, u, v)=\gamma_{1}(x, u, v)$ if and only if $\left[\mathbb{L}_{u} L_{x}, \mathbb{R}_{v} R_{[u \backslash(x v)]}\right]=I$ for all $x, u, v \in$ $Q$.

The proof of Theorem 2.1 can also be achieved by making use of Theorem 1.12. Take $a=u, b=\phi_{0}(x, u, v), c=u$ and $d=u \backslash(x v)$. Then, $\left(Q, \circ_{0}\right) \stackrel{I}{\cong}\left(Q, \circ_{1}\right)$ iff

1. $u \phi_{0}(x, u, v) \in N_{\mu}\left(\left(Q, \circ_{0}\right)\right)$,

3. $u \phi_{0}(x, u, v)=u[u \backslash(x v)] \Leftrightarrow$

2. $u[u \backslash(x v)] \in N_{\mu}\left(\left(Q, \circ_{0}\right)\right), \quad \mathcal{Q}$ is a Boolean group.

Theorem 2.2. Let $Q=(Q, \cdot, \backslash, /)$ be a universal Osborn loop. Then $\left(Q, \circ_{0}\right) \cong$ $\left(Q, \circ_{1}\right)$ if and only if there exists $(I, \beta, \gamma) \in A U T(Q)$ such that

$$
u v=x R_{v} \mathbb{L}_{u} \beta^{-1} L_{u} \mathbb{R}_{v} \cdot x R_{v} \mathbb{L}_{u}=x R_{v} \gamma^{-1} \mathbb{R}_{v} \cdot x R_{v} \mathbb{L}_{u}
$$

for all $x, u, v \in Q$.

Proof. Following Lemma $1.11,\left(Q, \circ_{0}\right) \cong\left(Q, \circ_{1}\right)$ if and only if there exists $(\alpha, \beta, \gamma) \in \operatorname{AUT}(\mathcal{Q})$ such that

$$
\begin{gathered}
\left(u, \phi_{0}(x, u, v), u \phi_{0}(x, u, v)\right)(\alpha, \beta, \gamma)=(u,[u \backslash(x v)], x v) \Leftrightarrow \\
\left(u \alpha, \phi_{0}(x, u, v) \beta,\left(u \phi_{0}(x, u, v)\right) \gamma\right)=(u,[u \backslash(x v)], x v) \Leftrightarrow \\
u \alpha=u, \phi_{0}(x, u, v) \beta=[u \backslash(x v)] \text { and }\left(u \phi_{0}(x, u, v)\right) \gamma=x v \Leftrightarrow \\
\alpha=I,\{u \backslash([(u v) /(u \backslash(x v))] v)\} \beta=u \backslash(x v) \text { and }\{[(u v) /(u \backslash(x v))] v\} \gamma=x v \Leftrightarrow \\
\alpha=I,[(u v) /(u \backslash(x v))] R_{v} \mathbb{L}_{u} \beta=x R_{v} \mathbb{L}_{u} \text { and }[(u v) /(u \backslash(x v))] R_{v} \gamma=x R_{v} \Leftrightarrow \\
\alpha=I,(u v) /(u \backslash(x v))=x R_{v} \mathbb{L}_{u} \beta^{-1} L_{u} \mathbb{R}_{v} \text { and }[(u v) /(u \backslash(x v))]=x R_{v} \gamma^{-1} \mathbb{R}_{v} \\
\Leftrightarrow \alpha=I, u v=x R_{v} \mathbb{L}_{u} \beta^{-1} L_{u} \mathbb{R}_{v} \cdot x R_{v} \mathbb{L}_{u} \text { and } u v=x R_{v} \gamma^{-1} \mathbb{R}_{v} \cdot x R_{v} \mathbb{L}_{u} \Leftrightarrow
\end{gathered}
$$

there exists $(I, \beta, \gamma) \in A U T(\mathcal{Q})$ such that

$$
u v=x R_{v} \mathbb{L}_{u} \beta^{-1} L_{u} \mathbb{R}_{v} \cdot x R_{v} \mathbb{L}_{u}=x R_{v} \gamma^{-1} \mathbb{R}_{v} \cdot x R_{v} \mathbb{L}_{u} .
$$

Remark 2.2. If the autotopism $(\alpha, \beta, \gamma)$ in Theorem 2.2 is the identity autotopism, then we shall have the equivalence of 1 . and 3 . of Theorem 2.1.

Corollary 2.3. Let $\mathcal{Q}=(Q, \cdot, \backslash, /)$ be a universal Osborn loop. Then $\left(Q, \circ_{0}\right) \cong$ $\left(Q, \circ_{1}\right)$ implies that there exists $(I, \beta, \gamma) \in A U T(2)$ such that $\gamma=\mathbb{L}_{u} \beta L_{u}$ for all $u \in Q$. Hence,

1. $\gamma=\beta$ iff $\left[\beta, L_{u}\right]=I$ or $\left[\gamma, L_{u}\right]=I$. Thence, $\beta$ is a $\rho$-regular permutation. 
2. $\gamma=L_{u}$ iff $\beta=L_{u}$. Thence, $\mathcal{Q}$ is an abelian group.

Proof. The proof of these follows from the fact in Theorem 2.2 that

$$
x R_{v} \mathbb{L}_{u} \beta^{-1} L_{u} \mathbb{R}_{v} \cdot x R_{v} \mathbb{L}_{u}=x R_{v} \gamma^{-1} \mathbb{R}_{v} \cdot x R_{v} \mathbb{L}_{u} \Rightarrow
$$

$\mathbb{L}_{u} \beta L_{u}=\gamma$ for all $u \in Q$

Theorem 2.4. Let $Q=(Q, \cdot, \backslash, /)$ be a universal Osborn loop. Then $\left(Q, *_{0}\right) \cong$ $\left(Q, *_{1}\right)$ if and only if there exists $(\delta, I, \pi) \in A U T(Q)$ such that

$$
u v=x \cdot x \delta R_{v} \mathbb{L}_{u}=x \cdot x R_{v} \pi \mathbb{L}_{u}
$$

for all $x, u, v \in Q$.

Proof. Following Lemma 1.11, $\left(Q, *_{0}\right) \cong\left(Q, *_{1}\right)$ if and only if there exists $(\delta, \varepsilon, \pi) \in \operatorname{AUT}(\mathcal{Q})$ such that $(x, v, x v)(\delta, \varepsilon, \pi)=\left(\phi_{1}(x, u, v), v, \phi_{1}(x, u, v) v\right)$. The procedure of the proof of the remaining part is similar to that of Theorem 2.2 .

Remark 2.3. If the autotopism $(\delta, \varepsilon, \pi)$ in Theorem 2.4 is the identity autotopism, then we shall have the equivalence of 2 . and 3 . of Theorem 2.1.

Corollary 2.5. Let $Q=(Q, \cdot, \backslash, /)$ be a universal Osborn loop. Then $\left(Q, *_{0}\right) \cong$ $\left(Q, *_{1}\right)$ implies that there exists $(\delta, I, \pi) \in A U T(\mathcal{Q})$ such that $\pi=\mathbb{R}_{v} \delta R_{v}$ for all $v \in Q$. Hence,

1. $\pi=\delta$ iff $\left[\delta, R_{v}\right]=I$ or $\left[\pi, R_{v}\right]=I$. Thence, $\delta$ is a $\lambda$-regular permutation.

2. $\delta=R_{v}$ iff $\pi=R_{v}$. Thence, $\mathcal{Q}$ is an abelian group.

Proof. The proof of these follows from the fact in Theorem 2.4 that

$$
x \cdot x \delta R_{v} \mathbb{L}_{u}=x \cdot x R_{v} \pi \mathbb{L}_{u} \Rightarrow
$$

$\pi=\mathbb{R}_{v} \delta R_{v}$ for all $v \in Q$

Theorem 2.6. Let $Q=(Q, \cdot, \backslash, /)$ be a universal Osborn loop. Then $\left(Q, \circ_{0}\right) \cong$ $\left(Q, \circ_{1}\right)$ and $\left(Q, *_{0}\right) \cong\left(Q, *_{1}\right)$ if and only if there exists $(I, \beta, \gamma),(\delta, I, \pi) \in$ $A U T(Q)$ such that

$$
\begin{gathered}
u v=x R_{v} \mathbb{L}_{u} \beta^{-1} L_{u} \mathbb{R}_{v} \cdot x R_{v} \mathbb{L}_{u}= \\
x R_{v} \gamma^{-1} \mathbb{R}_{v} \cdot x R_{v} \mathbb{L}_{u}=x \cdot x \delta R_{v} \mathbb{L}_{u}=x \cdot x R_{v} \pi \mathbb{L}_{u}
\end{gathered}
$$

for all $x, u, v \in Q$ 
Proof. This is achieved by simply combining Theorem 2.2 and Theorem 2.4.

Theorem 2.7. Let $Q=(Q, \cdot, \backslash, /)$ be a universal Osborn loop. If $\left(Q, \circ_{0}\right) \stackrel{\gamma_{01}^{\circ}}{\cong}$ $\left(Q, \circ_{1}\right)$ and $\left(Q, *_{0}\right) \stackrel{\gamma_{01}^{*}}{\cong}\left(Q, *_{1}\right)$, then $\gamma_{0} \gamma_{01}^{*} \gamma_{1}=\gamma_{01}^{\circ}$.

Proof. The commutative diagram in Equation 2.1 proves this.

Corollary 2.8. Let $Q=(Q, \cdot, \backslash, /)$ be a universal Osborn loop. If $\left(Q, \circ_{0}\right) \cong$ $\left(Q, \circ_{1}\right)$ and $\left(Q, *_{0}\right) \cong\left(Q, *_{1}\right)$, then the following are necessary and sufficient for each other.
1. $\beta=I$.
4. $\pi=I$.
6. $\left(Q, *_{0}\right) \stackrel{I}{\cong}\left(Q, *_{1}\right)$.
2. $\gamma=I$.
3. $\delta=I$.
5. $\left(Q, \circ_{0}\right) \stackrel{I}{\cong}\left(Q, \circ_{1}\right)$.
7. 2 is a boolean group.

Proof. To prove the equivalence of 1 . to 4 . and 7., use Equation 2.4 of Theorem 2.6. The proof of the equivalence of 5. to 7. follows from Theorem 2.1 .

Remark 2.4. Corollary 2.8 is a very important result in this study. It gives us the main distinctions between Theorem 2.1 and Theorem 2.6. That is, the necessary and sufficient condition(s) under which the isomorphisms $\left(Q, \circ_{0}\right) \cong\left(Q, \circ_{1}\right)$ and $\left(Q, *_{0}\right) \cong\left(Q, *_{1}\right)$ will be trivial. And the condition(s) is when any of the autotopic permutations of $\beta, \gamma, \delta$ and $\pi$ of Theorem 2.2 and Theorem 2.4 is equal to the identity mapping.

Next, it is important to deduce the actual definitions of the autotopic mappings $\beta, \gamma, \delta, \pi$ and the isomorphisms $\gamma_{01}^{*}$ and $\gamma_{01}^{\circ}$. Recall that by the necessary part of Lemma 1.11 , if $\mathcal{Q}=(Q, \cdot, \backslash, /)$ is a loop and $Q_{f, g} \stackrel{\ominus}{\cong}$ $Q_{c, d}$, then there exists $(A, B, C) \in A U T(Q)$ such that $(f, g, f g)(A, B, C)=$ $(c, d, c d)$. According to the proof of this,

$$
\begin{gathered}
(A, B, C)=\left(R_{g} \theta R_{d}^{-1}, L_{f} \theta L_{c}^{-1}, \theta\right) \Leftrightarrow \\
A=R_{g} \theta R_{d}^{-1}, B=L_{f} \theta L_{c}^{-1} \text { and } C=\theta .
\end{gathered}
$$

Thus,

$$
\begin{gathered}
I=\alpha=R_{\phi_{o}(x, u, v)} \gamma_{01}^{\circ} R_{[u \backslash(x v)]}^{-1}, \beta=L_{u} \gamma_{01}^{\circ} L_{u}^{-1} \text { and } \gamma=\gamma_{01}^{\circ} \\
\gamma_{01}^{\circ}=\mathbb{R}_{\phi_{o}(x, u, v)} R_{[u \backslash(x v)]}, \beta=L_{u} \mathbb{R}_{\phi_{o}(x, u, v)} R_{[u \backslash(x v)]} \mathbb{L}_{u}^{-1} \text { and } \\
\gamma=\mathbb{R}_{\phi_{o}(x, u, v)} R_{[u \backslash(x v)]}
\end{gathered}
$$


and

$$
\begin{gathered}
\delta=R_{v} \gamma_{01}^{*} R_{v}^{-1}, I=\varepsilon=L_{x} \gamma_{01}^{*} L_{\phi_{1}(x, u, v)}^{-1} \text { and } \pi=\gamma_{01}^{*} \\
\delta=R_{v} \gamma_{01}^{*} \mathbb{R}_{v}^{-1}, \gamma_{01}^{*}=\mathbb{L}_{x} L_{\phi_{1}(x, u, v)} \text { and } \pi=\gamma_{01}^{*} \\
\delta=R_{v} \mathbb{L}_{x} L_{\phi_{1}(x, u, v)} \mathbb{R}_{v}^{-1}, \gamma_{01}^{*}=\mathbb{L}_{x} L_{\phi_{1}(x, u, v)} \text { and } \pi=\mathbb{L}_{x} L_{\phi_{1}(x, u, v)} .
\end{gathered}
$$

Therefore, Theorem 2.2 and Theorem 2.4 can now be restated as follows.

Theorem 2.9. Let $\mathcal{Q}=(Q, \cdot, \backslash, /)$ be a universal Osborn loop. Then $\left(Q, \circ_{0}\right) \stackrel{\gamma_{01}^{\circ}}{\cong}$ $\left(Q, \circ_{1}\right)$ if and only if

$$
y \cdot u \backslash\left[(u z) \psi_{0}\right]=(y z) \psi_{0} \text { and } u v=x R_{v}\left(R_{v} \psi_{0}\right)^{-1} \cdot x R_{v} \mathbb{L}_{u}
$$

where $\psi_{0}=\mathbb{R}_{\phi_{o}(x, u, v)} R_{[u \backslash(x v)]}$ for all $x, y, z, u, v \in Q$

Proof. Simply substitute

$$
\beta=L_{u} \mathbb{R}_{\phi_{o}(x, u, v)} R_{[u \backslash(x v)]} \mathbb{L}_{u}^{-1} \text { and } \gamma=\mathbb{R}_{\phi_{o}(x, u, v)} R_{[u \backslash(x v)]}
$$

into Equation 2.2 of Theorem 2.2.

Theorem 2.10. Let $\mathcal{Q}=(Q, \cdot, \backslash, /)$ be a universal Osborn loop. Then $\left(Q, *_{0}\right) \stackrel{\gamma_{01}^{*}}{\cong}\left(Q, *_{1}\right)$ if and only if

$$
\left[(y v) \psi_{1}\right] / v \cdot z=(y z) \psi_{1} \text { and } u v=x \cdot u \backslash\left[(x v) \psi_{1}\right]
$$

where $\psi_{1}=\mathbb{L}_{x} L_{\phi_{1}(x, u, v)}$ for all $x, y, z, u, v \in Q$

Proof. Simply substitute

$$
\delta=R_{v} \mathbb{L}_{x} L_{\phi_{1}(x, u, v)} \mathbb{R}_{v}^{-1} \text { and } \pi=\mathbb{L}_{x} L_{\phi_{1}(x, u, v)}
$$

into Equation 2.3 of Theorem 2.4.

Lemma 2.11. Let $\mathcal{Q}=(Q, \cdot, \backslash, /)$ be a loop.

1. 2 is a universal Osborn loop and obeys Equation 2.6 if and only if $\gamma_{0}, \gamma_{01}^{\circ} \in B S_{2}(\mathcal{Q})$.

2. 2 is a universal Osborn loop and obeys Equation 2.7 if and only if $\gamma_{1}, \gamma_{01}^{*} \in B S_{2}(2)$.

Proof. This follows by combining Theorem 1.7, Theorem 1.8, Theorem 2.2 and Theorem 2.4

Remark 2.5. It is a self exercise to confirm if $\left(Q, \circ_{0}\right) \stackrel{\gamma_{01}^{\circ}}{\cong}\left(Q, \circ_{1}\right)$ and $\left(Q, *_{0}\right) \stackrel{\gamma_{01}^{*}}{\cong}$ $\left(Q, *_{1}\right)$ in some universal Osborn loops like Moufang loops and extra loops by simply verifying Equation 2.6 and Equation 2.7. Furthermore, the relation $\gamma_{0} \gamma_{01}^{*} \gamma_{1}=\gamma_{01}^{\circ}$ of Theorem 2.7 is justifiable as well. It must be noted also, that in any universal Osborn loop Q, Equation 2.6 and Equation 2.7 are necessary and sufficient conditions for $\gamma_{01}^{*}, \gamma_{01}^{\circ} \in B S_{2}(\mathcal{Q})$. 
By combining the commutative diagrams in Equation 1.5 and Equation 1.6, we have the commutative diagram below.

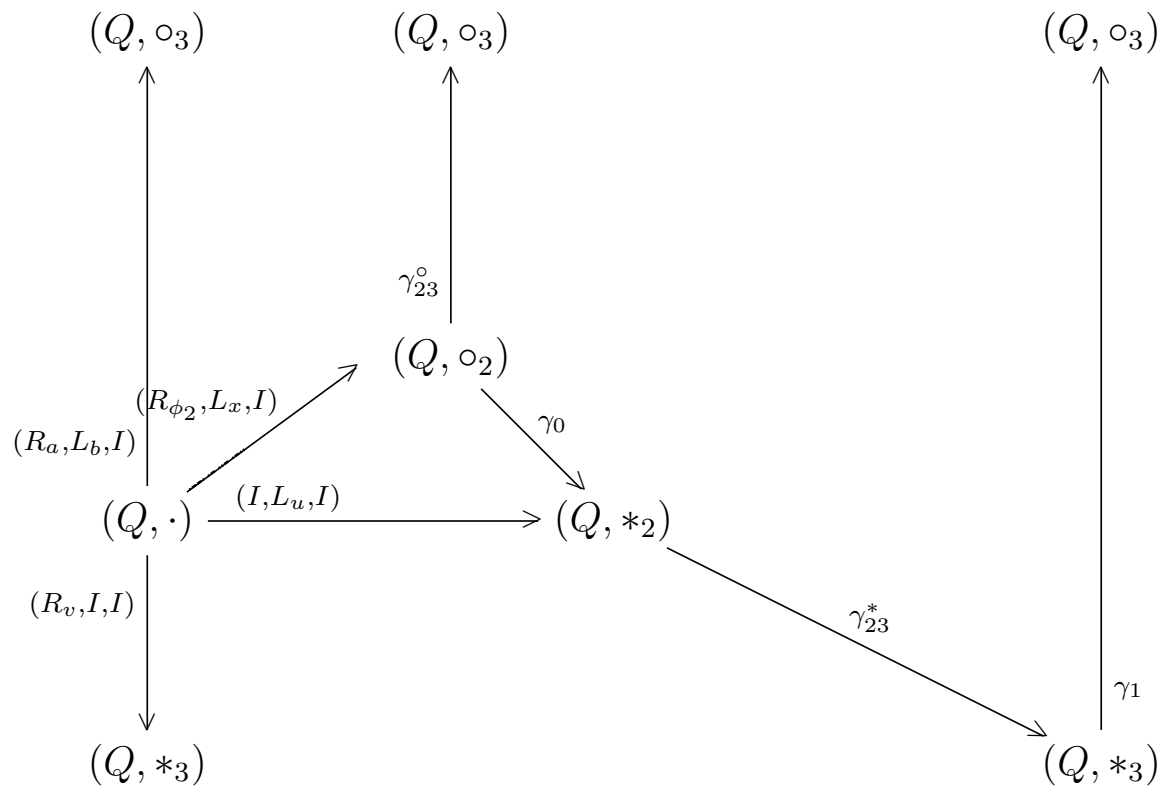

where $a=[u \backslash(x v)]$ and $b=\{[x \cdot u \backslash v] / v\}$.

Theorem 2.12. Let $\mathcal{Q}=(Q, \cdot, \backslash, /)$ be a universal Osborn loop. Then $\left(Q, \circ_{2}\right) \cong\left(Q, \circ_{3}\right)$ if and only if there exists $(\lambda, \mu, \nu) \in A U T(Q)$ such that

$$
\lambda=R_{u \backslash v} \mathbb{R}_{v}, \mu=L_{u} \mathbb{L}_{u \backslash v} \text { and }\left[x \cdot x R_{v} \mathbb{L}_{u} \mu^{-1}\right] \nu=x \lambda \cdot x R_{v} \mathbb{L}_{u}
$$

for all $x, u, v \in Q$.

Proof. Following Lemma $1.11,\left(Q, \circ_{2}\right) \cong\left(Q, \circ_{3}\right)$ if and only if there exists $(\lambda, \mu, \nu) \in A U T(Q)$ such that $\left(x, \phi_{2}(x, u, v), x \phi_{2}(x, u, v)\right)(\lambda, \mu, \nu)=([x$. $u \backslash v] / v,[u \backslash(x v)],\{[x \cdot u \backslash v] / v\}[u \backslash(x v)])$. The procedure of the proof of the remaining part is similar to that of Theorem 2.2.

Lemma 2.13. Let $Q=(Q, \cdot, \backslash, /)$ be a universal Osborn loop. Then $\left(Q, \circ_{2}\right) \stackrel{\gamma_{23}^{\circ}}{\cong}$ $\left(Q, \circ_{3}\right)$ if and only if there exists $\left(\lambda, \mu, \gamma_{23}^{\circ}\right) \in A U T(Q)$ such that

$$
\begin{gathered}
\gamma_{23}^{\circ}=\mathbb{R}_{\phi_{2}(x, u, v)} R_{u \backslash v} \mathbb{R}_{v} R_{[u \backslash(x v)]}=\mathbb{L}_{x} L_{u} \mathbb{L}_{u \backslash v} L_{\{[x \cdot u \backslash v] / v\}} \text { and } \\
{\left[x \cdot x R_{v} \mathbb{L}_{u} \mu^{-1}\right] \gamma_{23}^{\circ}=x \lambda \cdot x R_{v} \mathbb{L}_{u}}
\end{gathered}
$$

for all $x, u, v \in Q$.

Proof. Considering the commutative diagram in Equation 2.8 and using Equation 2.5,

$$
\lambda=R_{\phi_{2}(x, u, v)} \gamma_{23}^{\circ} R_{[u \backslash(x v)]}^{-1}, \quad \mu=L_{x} \gamma_{23}^{\circ} L_{\{[x \cdot u \backslash v] / v\}}^{-1} \text { and } \nu=\gamma_{23}^{\circ} .
$$

The final conclusion follows from Theorem 2.12. 
Corollary 2.14. Let $\mathcal{Q}=(Q, \cdot, \backslash, /)$ be a universal Osborn loop. $\gamma_{23}^{\circ} \in$ $B S_{2}(\mathcal{Q})$ if and only if there exists $\left(\lambda, \mu, \gamma_{23}^{\circ}\right) \in A U T(\mathcal{Q})$ such that

$$
\begin{gathered}
\gamma_{23}^{\circ}=\mathbb{R}_{\phi_{2}(x, u, v)} R_{u \backslash v} \mathbb{R}_{v} R_{[u \backslash(x v)]}=\mathbb{L}_{x} L_{u} \mathbb{L}_{u \backslash v} L_{\{[x \cdot u \backslash v] / v\}} \text { and } \\
{\left[x \cdot x R_{v} \mathbb{L}_{u} \mu^{-1}\right] \gamma_{23}^{\circ}=x \lambda \cdot x R_{v} \mathbb{L}_{u}}
\end{gathered}
$$

for all $x, u, v \in Q$.

Proof. This follows from Lemma2.13.

Corollary 2.15. Let $\mathcal{Q}=(Q, \cdot, \backslash, /)$ be a loop. $\mathcal{Q}$ is a universal Osborn loop and $\gamma_{23}^{\circ} \in B S_{2}(\mathcal{Q})$ implies $\gamma_{0} \in B S_{2}(\mathcal{Q})$ and there exists $\left(\lambda, \mu, \gamma_{23}^{\circ}\right) \in A U T(\mathcal{Q})$ such that

$$
\begin{gathered}
\gamma_{23}^{\circ}=\mathbb{R}_{\phi_{2}(x, u, v)} R_{u \backslash v} \mathbb{R}_{v} R_{[u \backslash(x v)]}=\mathbb{L}_{x} L_{u} \mathbb{L}_{u \backslash v} L_{\{[x \cdot u \backslash v] / v\}} \text { and } \\
{\left[x \cdot x R_{v} \mathbb{L}_{u} \mu^{-1}\right] \gamma_{23}^{\circ}=x \lambda \cdot x R_{v} \mathbb{L}_{u}}
\end{gathered}
$$

for all $x, u, v \in Q$.

Proof. This follows from Theorem 1.9 and Lemma2.13.

\section{Simplicial Complex of Isotopes of a Universal Osborn Loop}

Theorem 2.16. Let $(Q, \cdot)$ be a loop. Let $V_{0}(Q)=\left\{(Q, \cdot),\left(Q, \circ_{0}\right),\left(Q, *_{0}\right)\right\}$ and $S_{0}(Q)=\left\{\{(Q, \cdot)\},\left\{\left(Q, \circ_{0}\right)\right\},\left\{\left(Q, *_{0}\right)\right\},\left\{\left(Q, \circ_{0}\right),\left(Q, *_{0}\right)\right\}\right\}$. Then, $(Q, \cdot)$ is a universal Osborn loop if and only if $K_{0}(Q)=\left(V_{0}(Q), S_{0}(Q)\right)$ is a simplicial complex of isotopes of $(Q, \cdot)$.

Proof. This is proved with the help of Theorem 1.7.

Theorem 2.17. Let $(Q, \cdot)$ be a loop. Let $V_{1}(Q)=\left\{(Q, \cdot),\left(Q, \circ_{1}\right),\left(Q, *_{1}\right)\right\}$ and $S_{1}(Q)=\left\{\{(Q, \cdot)\},\left\{\left(Q, \circ_{1}\right)\right\},\left\{\left(Q, *_{1}\right)\right\},\left\{\left(Q, \circ_{1}\right),\left(Q, *_{1}\right)\right\}\right\}$. Then, $(Q, \cdot)$ is a universal Osborn loop if and only if $K_{1}(Q)=\left(V_{1}(Q), S_{1}(Q)\right)$ is a simplicial complex of isotopes of $(Q, \cdot)$.

Proof. This is proved with the help of Theorem 1.8.

Theorem 2.18. Let $(Q, \cdot)$ be a loop. Let $V_{2}(Q)=\left\{(Q, \cdot),\left(Q, \circ_{2}\right),\left(Q, *_{2}\right)\right\}$ and $S_{2}(Q)=\left\{\{(Q, \cdot)\},\left\{\left(Q, \circ_{2}\right)\right\},\left\{\left(Q, *_{2}\right)\right\},\left\{\left(Q, \circ_{2}\right),\left(Q, *_{2}\right)\right\}\right\}$. If $(Q, \cdot)$ is a universal Osborn loop, then $K_{2}(Q)=\left(V_{2}(Q), S_{2}(Q)\right)$ is a simplicial complex of isotopes of $(Q, \cdot)$. 
Proof. This is proved with Theorem 1.9.

Theorem 2.19. Let $(Q, \cdot)$ be a loop. Let $V_{3}(Q)=\left\{(Q, \cdot),\left(Q, \circ_{3}\right),\left(Q, *_{3}\right)\right\}$ and $S_{3}(Q)=\left\{\{(Q, \cdot)\},\left\{\left(Q, \circ_{3}\right)\right\},\left\{\left(Q, *_{3}\right)\right\},\left\{\left(Q, \circ_{3}\right),\left(Q, *_{3}\right)\right\}\right\}$. If $(Q, \cdot)$ is a universal Osborn loop, then $K_{3}(Q)=\left(V_{3}(Q), S_{3}(Q)\right)$ is a simplicial complex of isotopes of $(Q, \cdot)$.

Proof. This is proved with the aid of Theorem 1.10.

Corollary 2.20. Let $(Q, \cdot)$ be a loop. Let $V_{i}(Q)=\left\{(Q, \cdot),\left(Q, \circ_{i}\right),\left(Q, *_{i}\right)\right\}$ and $S_{i}(Q)=\left\{\{(Q, \cdot)\},\left\{\left(Q, \circ_{i}\right)\right\},\left\{\left(Q, *_{i}\right)\right\},\left\{\left(Q, \circ_{i}\right),\left(Q, *_{i}\right)\right\}\right\}$ for $i=0,1$. Then, $(Q, \cdot)$ is a universal Osborn loop if and only if $K_{01}(Q)=K_{0}(Q) \cup$ $K_{1}(Q)=\left(V_{0}(Q) \cup V_{1}(Q), S_{0}(Q) \cup S_{1}(Q)\right)$ is a simplicial complex of isotopes of $(Q, \cdot)$.

Proof. This follows from Theorem 2.16 and Theorem 2.17.

Corollary 2.21. Let $(Q, \cdot)$ be a loop. Let $V_{i}(Q)=\left\{(Q, \cdot),\left(Q, \circ_{i}\right),\left(Q, *_{i}\right)\right\}$ and $S_{i}(Q)=\left\{\{(Q, \cdot)\},\left\{\left(Q, \circ_{i}\right)\right\},\left\{\left(Q, *_{i}\right)\right\},\left\{\left(Q, \circ_{i}\right),\left(Q, *_{i}\right)\right\}\right\}$ for $i=2,3$. If $(Q, \cdot)$ is a universal Osborn loop, then $K_{23}(Q)=K_{2}(Q) \cup K_{3}(Q)=\left(V_{2}(Q) \cup\right.$ $\left.V_{3}(Q), S_{2}(Q) \cup S_{3}(Q)\right)$ is a simplicial complex of isotopes of $(Q, \cdot)$.

Proof. This follows from Theorem 2.18 and Theorem 2.19.

Corollary 2.22. Let $(Q, \cdot)$ be a loop. Let $V_{i}(Q)=\left\{(Q, \cdot),\left(Q, \circ_{i}\right),\left(Q, *_{i}\right)\right\}$ and $S_{i}(Q)=\left\{\{(Q, \cdot)\},\left\{\left(Q, \circ_{i}\right)\right\},\left\{\left(Q, *_{i}\right)\right\},\left\{\left(Q, \circ_{i}\right),\left(Q, *_{i}\right)\right\}\right\}$ for $i=0,1,2,3$. If $(Q, \cdot)$ is a universal Osborn loop, then

$K_{0123}(Q)=\bigcup_{i=0}^{3} K_{i}(Q)=\left(\bigcup_{i=0}^{3} V_{i}(Q), \bigcup_{i=0}^{3} S_{i}(Q)\right)$ is a simplicial complex of isotopes of $(Q, \cdot)$.

Proof. This is proved by combining Corollary 2.20 and Corollary 2.21.

Theorem 2.23. Let $(Q, \cdot)$ be a loop. Let $V_{01}(Q)=\left\{(Q, \cdot),\left(Q, \circ_{0}\right),\left(Q, *_{0}\right)\right.$, $\left.\left(Q, \circ_{1}\right),\left(Q, *_{1}\right)\right\}$ and $S_{10}(Q)=\left\{\{(Q, \cdot)\},\left\{\left(Q, \circ_{0}\right)\right\},\left\{\left(Q, *_{0}\right)\right\},\left\{\left(Q, \circ_{1}\right)\right\}\right.$, $\left\{\left(Q, *_{1}\right)\right\},\left\{\left(Q, \circ_{0}\right),\left(Q, *_{0}\right)\right\},\left\{\left(Q, \circ_{1}\right),\left(Q, *_{1}\right)\right\},\left\{\left(Q, \circ_{0}\right),\left(Q, \circ_{1}\right)\right\},\left\{\left(Q, *_{0}\right)\right.$, $\left.\left(Q, *_{1}\right)\right\},\left\{\left(Q, \circ_{0}\right),\left(Q, *_{1}\right)\right\},\left\{\left(Q, \circ_{1}\right),\left(Q, *_{0}\right)\right\},\left\{\left(Q, \circ_{0}\right),\left(Q, \circ_{1}\right),\left(Q, *_{0}\right)\right\}$, 
$\left\{\left(Q, \circ_{0}\right),\left(Q, \circ_{1}\right),\left(Q, *_{1}\right)\right\},\left\{\left(Q, *_{0}\right),\left(Q, *_{1}\right),\left(Q, \circ_{0}\right)\right\}$,

$\left.\left\{\left(Q, *_{0}\right),\left(Q, *_{1}\right),\left(Q, \circ_{1}\right)\right\},\left\{\left(Q, \circ_{0}\right),\left(Q, \circ_{1}\right),\left(Q, *_{0}\right),\left(Q, *_{1}\right)\right\}\right\}$. Then, $(Q, \cdot)$ is a universal Osborn loop and obey Equation 2.6 and Equation 2.7 if and only if $K_{10}(Q)=\left(V_{01}(Q), S_{10}(Q)\right)$ is a simplicial complex of isotopes of $(Q, \cdot)$.

Proof. This is proved with the aid of Theorem 2.16, Theorem 2.17, Theorem 2.9 and Theorem 2.10.

Theorem 2.24. Let $(Q, \cdot)$ be a universal Osborn loop.

$$
\begin{aligned}
& \text { Let } V_{i}(Q)=\left\{(Q, \cdot),\left(Q, \circ_{i}\right),\left(Q, *_{i}\right)\right\} \text {, } \\
& S_{i}(Q)=\left\{\{(Q, \cdot)\},\left\{\left(Q, \circ_{i}\right)\right\},\left\{\left(Q, *_{i}\right)\right\},\left\{\left(Q, \circ_{i}\right),\left(Q, *_{i}\right)\right\}\right\} \text { and } \\
& K_{i}=\left(V_{i}(Q), S_{i}(Q)\right) \text { for } i=0,1,2,3 \text {. Define } f_{i j}: K_{i} \rightarrow K_{j} \text { as } \\
& f_{i j}: \begin{cases}(Q, \cdot) & \longmapsto(Q, \cdot) \\
\left(Q, \circ_{i}\right) & \longmapsto\left(Q, \circ_{j}\right) \quad i, j=0,1,2,3 \text { such that } i \neq j . \\
\left(Q, *_{i}\right) & \longmapsto\left(Q, *_{j}\right)\end{cases}
\end{aligned}
$$

Then, $f_{i j}$ is a simplicial map.

Proof. This is proved by Theorem 2.16, Theorem 2.17, Theorem 2.18 and Theorem 2.19.

Theorem 2.25. Let $(G, \cdot)$ and $(H, \star)$ be two loop isotopes under the triple $(A, B, C)$. For $D \in\{A, B, C\}$, if $D=E_{1} E_{2} \cdots E_{i} \cdots E_{n}, E_{i}: G \rightarrow$ $H, i=1, \cdots n$ been bijections such that there does not exist $r \geq n$ for which $D=E_{1} E_{2} \cdots E_{i} \cdots E_{r}$, then the length of $D,|D|=n$ units. If $D=I$, the identity mapping, then $|D|=0$. The length of the isotopism $(G, \cdot) \underset{\text { Isotopism }}{\stackrel{(A, B, C)}{\longrightarrow}}(H, \star)$ is giving by $|(A, B, C)|=|A|+|B|+|C|$ units. For an isotopism $(G, \cdot) \underset{\text { Isotopism }}{\stackrel{(A, B, C)}{\longrightarrow}}(H, \star)$, let the two loops $(G, \cdot)$ and $(H, \star)$ represent points in a 3-dimensional space and let an isotopism from $(G, \cdot)$ to $(H, \star)$ be a line with $(G, \cdot)$ and $(H, \star)$ as end-points. The set of loops $V_{01}(Q)=$ $\left\{(Q, \cdot),\left(Q, \circ_{0}\right),\left(Q, *_{0}\right),\left(Q, \circ_{1}\right),\left(Q, *_{1}\right)\right\}$ where $(Q, \cdot)$ is a universal Osborn loop, form a rectangular pyramid with apex $(Q, \cdot)$.

Proof. We shall make use of the combined commutative diagram (2.1) as shown in the proof of Theorem 2.1. There are four isotopes of $(Q, \cdot)$ as shown in the combined commutative diagram $(2.1)$, namely $\left(Q, \circ_{i}\right),\left(Q, *_{i}\right)$ for $i=0,1$. The length of each of the isotopisms $\left(R_{[u \backslash(x v)]}, L_{u}, I\right),\left(R_{\phi_{0}}, L_{u}, I\right)$, $\left(R_{v}, L_{\phi_{1}}, I\right),\left(R_{v}, L_{x}, I\right)$ is 2 units. The length of each of the isomorphisms 
$\gamma_{0}(x, u, v)=\mathbb{R}_{v} R_{[u \backslash(x v)]} \mathbb{L}_{u} L_{x}$ and $\gamma_{1}(x, u, v)=\mathbb{R}_{v} R_{[u \backslash(x v)]} \mathbb{L}_{u} L_{x}$ is 12 units. The length of each of the isomorphisms $\gamma_{01}^{\circ}=\mathbb{R}_{\phi_{o}(x, u, v)} R_{[u \backslash(x v)]}$ and $\gamma_{01}^{*}=$ $\mathbb{L}_{x} L_{\phi_{1}(x, u, v)}$ is 6 units. Hence, the four loop isotopes $\left(Q, \circ_{i}\right),\left(Q, *_{i}\right)$ for $i=$ 0,1 of $(Q, \cdot)$ form a rectangle. Thus, taking $(Q, \cdot)$ as an apex and the four isotopism as lines drawn from the apex to the four vertices of the rectangle, we have a rectangular pyramid.

\section{References}

[1] B. F. Bryant and H. Schneider, Principal loop-isotopes of quasigroups, Canad. J. Math., 18, (1966), 120-125

[2] A. A. Drisko, Loops with transitive automorphisms, J. Algebra, 184, (1966), 213-229

[3] T. G. Jaiyéọlá, J. O. Adéníran, and A. R. T. Sòlárìn, The universality of Osborn loops, Acta Universitatis Apulensis Mathematics-Informatics, 26, (2011), 301-320

[4] T. G. Jaiyéọlá, Osborn loops and their universality, Scientific Annals of "Al.I. Cuza" University of Iasi., 58, (2012), 437-452

[5] S. K. Stein, On the foundations of quasigroups, Trans. Amer. Math. Soc., 85, (1957), $228-256$

Jaíyéọlá Tèmítọ́pẹ́ Gbọ́láhàn

Department of Mathematics

Faculty of Science,

Obafemi Awolowo University,

Ile Ife,

Nigeria.

E-mail: jaiyeolatemitope@yahoo.com, tjayeola@oauife.edu.ng

Received: 22.05.2014

Accepted: 30.06 .2014 